\title{
Modeling and Optimization of SUV Based on EuroNCAP Pedestrian Head Protection
}

\author{
XING Yanyun ${ }^{1,2, a}$, BIAN Xueliang ${ }^{1, b}$, LIU Xuelong ${ }^{3, c}$, HUANG Senren ${ }^{3, \mathrm{e}}$, YU \\ $\mathrm{Bo}^{3, \mathrm{e}}$
}

${ }^{1}$ College of Mechanical Engineering, Hebei University of Technology, TianJin, China, 300401 ${ }^{2}$ College of Automotive and Transportation Tianjin University of Technology and Education, China, TianJin, 300222,

${ }^{3}$ Automobile Engineering Research Institute China Automotive Technology and Research Center, Tianjin, China, 300162

axyyyb2014@163.com, bjluqicheyb@hotmail.com, ${ }^{c} 148087009 @ 163 . c o m,{ }^{d} h s r e n 123 @ 163 . c o m$, eyuboxyy@163.com

Keywords: Modeling and Optimization,SUV, EuroNCAP

Abstract. In this paper, the pedestrian head impact protection performance of SUV is evaluated base on European New Car Assessment Program (EuroNCAP) pedestrian protection five star criterion of 2013 edition. The front body of CAE model was built according to EuroNCAP , the model was correlated very well with the test based on the data of acceleration. In order to improve the performance for pedestrian head impact, the optimization proposal was provided after analyzing the results. It is showed that the pedestrian head impact performance of the SUV was enhanced obviously after the optimization implemented, the result also can be a guideline for vehicle pedestrian head performance improvement.

\section{Introduction}

European New Car Assessment Program (EuroNCAP) is one of the car safety authority assessment institution recognized in the world. It is one of the important automobile design goals to obtain high star-rated institution of EuroNCAP. A comprehensive assessment of EuroNCAP safety protection includes adult, child protection, pedestrian protection and auxiliary safety measure. If one models want to get the corresponding star, their scores of each term must reach the lower limit value. And the lower limit value increases year by year, especially the pedestrian protection threshold. To achieve 2013 version EuroNCAP 5 star level, pedestrian protection term score must reach more than $60 \%$ of the total 36 points, which is 21.6 points, and the corresponding point of the simulation calculation results shall be supplied to EuroNCAP official.

In this paper, with the EuroNCAP 5 star level as the goal, we analyze and evaluate the pedestrian head protection performance of one SUV and optimized the structure. The design method and key factors that influence the performance of pedestrian head protection, are discussed and proposed, such as the front vehicle shape, local structural design etc. 


\section{Experiment and results evaluation of pedestrian head protection}

In this paper, the experiments about the head, thighs and cruses were carried out firstly based on the evaluation standard of EuroNCAP2010 version, the experiment results are the basis reference for the subsequent simulation, experiment and results related to head as shown in fig.1.

Four adult head model and eight children head model impact points are selected, which are named respectively, $\mathrm{AH} 1, \mathrm{AH} 2, \mathrm{AH} 3, \mathrm{AH} 4$, and $\mathrm{CH} 1, \mathrm{CH} 2, \mathrm{CH} 3, \mathrm{CH} 4, \mathrm{CH} 5, \mathrm{CH} 6, \mathrm{CH}$, $\mathrm{CH} 8$, the test results are shown in table 1.

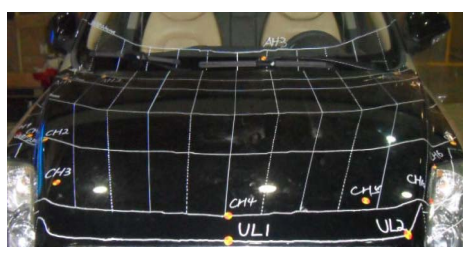

Fig. 1 head mould test score and crash location diagram

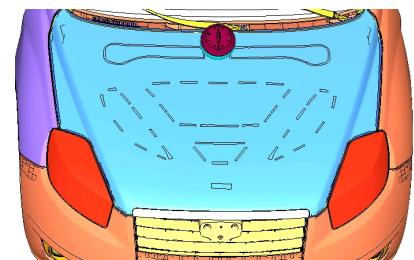

Fig. 2 schematic diagram of finite element model

Table 1 crash experiment results

\begin{tabular}{|c|c|c|c|c|}
\hline NO. & impact point and coordinates & Impact velocity $(\mathrm{km} / \mathrm{h})$ & impact angle & HIC15 \\
\hline 1 & AH1(171.44,-152.11,748.97) & 40.06 & $65^{\circ}$ & 1554.4 \\
\hline 2 & $\mathrm{AH} 2(139.45,-314.63,760.93)$ & 39.8 & $65^{\circ}$ & 974 \\
\hline 3 & $\mathrm{AH} 3(295.91,-936.89,860.09)$ & 40.04 & $65^{\circ}$ & 1836.6 \\
\hline 4 & $\mathrm{AH} 4(230.05,-1467.05,763.07)$ & 39.099 & $65^{\circ}$ & 1927.8 \\
\hline 5 & $\mathrm{CH} 1(-220.51,-150.99,646.95)$ & 39.97 & $50^{\circ}$ & 892.4 \\
\hline 6 & $\mathrm{CH} 2(-350.85,-249.23,667.73)$ & 40.51 & $50^{\circ}$ & 639.6 \\
\hline 7 & $\mathrm{CH} 3(-583.04,-359.93,623.29)$ & 40.05 & $50^{\circ}$ & 1150.1 \\
\hline 8 & $\mathrm{CH} 4(-726.25,-836.4,598.34)$ & 40.28 & $50^{\circ}$ & 1082 \\
\hline 9 & $\mathrm{CH} 5(-655.88,-1185.84,609.63)$ & 40.92 & $50^{\circ}$ & 1514 \\
\hline 10 & $\mathrm{CH} 6(-393.7,-1439.19,613.81)$ & 40.59 & $50^{\circ}$ & 791 \\
\hline 11 & $\mathrm{CH} 7(-239.3,-1549.82,636.87)$ & 40.05 & $50^{\circ}$ & 1546.3 \\
\hline 12 & $\mathrm{CH} 8(114.57,-1504.76)$ & 40.53 & $50^{\circ}$ & 1229 \\
\hline
\end{tabular}

From the experimental results, we can see that the HIC15 of adult impact windshield points are above 1700, children's test points 7, 8, 9, 12 HIC15values are greater than 1000, body structure need to be further optimized for the target of EuroNCAP pedestrian protection 5 star-rated.

\section{Structure modeling and validation of dynamic simulation}

The front parts of A pillar which have great influence to the head injury are remained, including engine hood and its affiliated parts, lights, wine, water tank and radiator, front bumper assembly, windshield, etc, model shown in figure 2, the freedom for six directions is forbidden in the joint of the cutting face or the joint of body and sub-frame.

Head collision results are greatly influenced by partial car body structure and the location of head model. The coordinate origin using in crash test is not consistent with the origin using in body coordinate system, the origin position using in the test is shown in figure 3 and in table 2 , and the coordinates switch between test and the finite element model is shown in Table 3. 


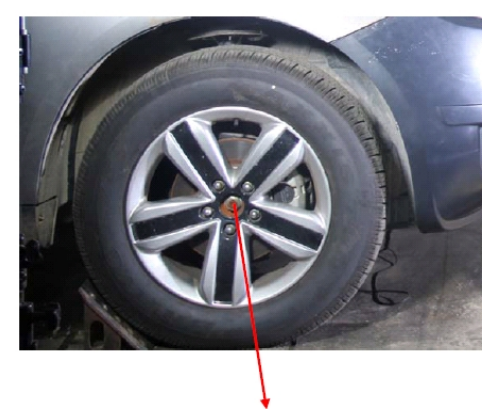

Fig. 3 test origin of coordinates
Table 2 the test vehicle state

\begin{tabular}{|c|c|}
\hline tyre pressure & $0.22 \mathrm{MPa}$ \\
\hline Kerb mass & $1.512 \mathrm{t}$ \\
\hline the height of left front wheel-housing & $805 \mathrm{~mm}$ \\
\hline the height of right front wheel-housing & $800 \mathrm{~mm}$ \\
\hline the height of left rear wheel-housing & $798 \mathrm{~mm}$ \\
\hline the height of right rear wheel-housing & $798 \mathrm{~mm}$ \\
\hline Ground height & 340. \\
& $03 \mathrm{~mm}$ \\
\hline
\end{tabular}

Table 3 conversion tables between test and the finite element

\begin{tabular}{|c|c|c|c|c|c|c|}
\hline Position & X(Test) & Y(Test) & Z(Test) & X(FEM) & Y(FEM) & Z(FEM) \\
\hline AH1 & 171.44 & -152.11 & 748.97 & 171.89 & 701.25 & 722.37 \\
\hline AH2 & 139.45 & -314.63 & 760.93 & 139.9 & 538.73 & 734.33 \\
\hline AH3 & 295.91 & -936.89 & 806.09 & 296.36 & -83.53 & 779.49 \\
\hline AH4 & 230.05 & -1467.05 & 763.07 & 230.5 & -613.69 & 736.47 \\
\hline CH1 & -220.51 & -150.99 & 646.95 & -220.06 & 702.37 & 620.35 \\
\hline CH2 & -350.85 & -249.23 & 667.73 & -350.4 & 604.13 & 641.13 \\
\hline CH3 & -583.04 & -359.93 & 623.29 & -582.59 & 493.43 & 596.69 \\
\hline CH4 & -726.25 & -836.4 & 598.34 & -725.8 & 16.96 & 571.74 \\
\hline CH5 & -655.88 & -1185.84 & 609.63 & -655.43 & -332.48 & 583.03 \\
\hline CH6 & -393.7 & -1439.19 & 613.81 & -393.25 & -585.83 & 587.21 \\
\hline CH7 & -239.3 & -1549.82 & 636.87 & -238.85 & -696.46 & 610.27 \\
\hline CH8 & 114.57 & -1504.76 & 743.11 & 115.02 & -651.4 & 716.51 \\
\hline
\end{tabular}

According to the test location and results, $\mathrm{CH} 2, \mathrm{CH} 3, \mathrm{CH} 4, \mathrm{CH} 5, \mathrm{AH} 1, \mathrm{AH} 4$ is selected as the target point to contrast, the comparison results is shown in figure4. Mat32 in Dyna materials database is assigned to glass as material parameters, and the rupture failure is ignored in the simulation.
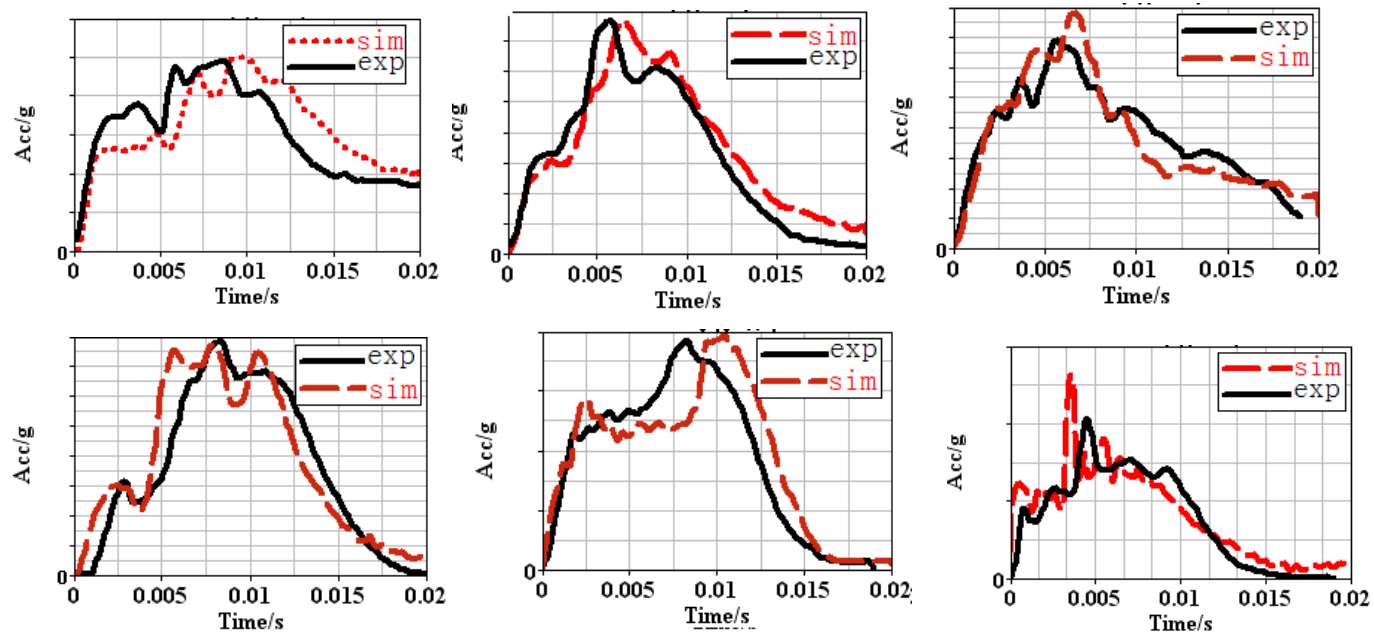

Fig. 4 comparison result respective about $\mathrm{CH} 2, \mathrm{CH} 3, \mathrm{CH} 4, \mathrm{CH} 5, \mathrm{AH} 1$ and $\mathrm{AH} 4$ 


\begin{tabular}{|c|c|c|c|c|c|c|c|}
\hline $\begin{array}{c}\text { HIC } \\
\text { POS }\end{array}$ & TEST & FEM & $\begin{array}{c}\text { Tolerance } \\
\text { rate }\end{array}$ & $\begin{array}{c}\text { TIC } \\
\text { POS }\end{array}$ & TEST & FEM & $\begin{array}{c}\text { Tolerance } \\
\text { rate }\end{array}$ \\
\hline CH2 & 640 & 653 & $2 \%$ & CH5 & 1513 & 1599 & $1.2 \%$ \\
\hline CH3 & 1150 & 1196 & $4 \%$ & AH1 & 1554 & 1517 & $2.4 \%$ \\
\hline CH4 & 1104 & 1082 & $1.9 \%$ & AH4 & 1927 & 1937 & $0.5 \%$ \\
\hline
\end{tabular}

According to the evaluation of head acceleration curve and HIC15, we can see the impacting finite element model could reflect the structural characteristics of test vehicle, and the simulation model is effective and reliable. So it can be used to optimize and improve the body structure about pedestrian head protection.

\section{Analysis and improvement the structure for head impact regional}

According to the 2013 edition of the EuroNCAP pedestrian protection for head evaluation standard marking requirements, the test WAD lines, front end test impact test points are divided with the CAVA tools and Hyperworks, as shown in figure 5.

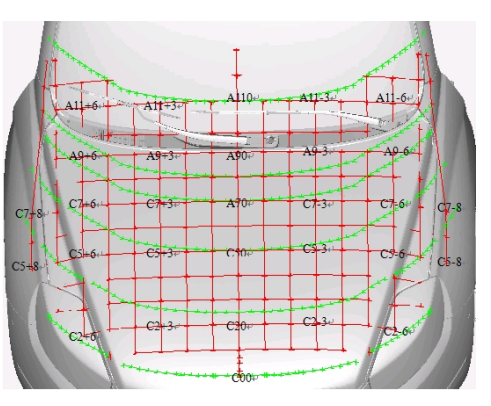

Fig.5 the test WAD lines

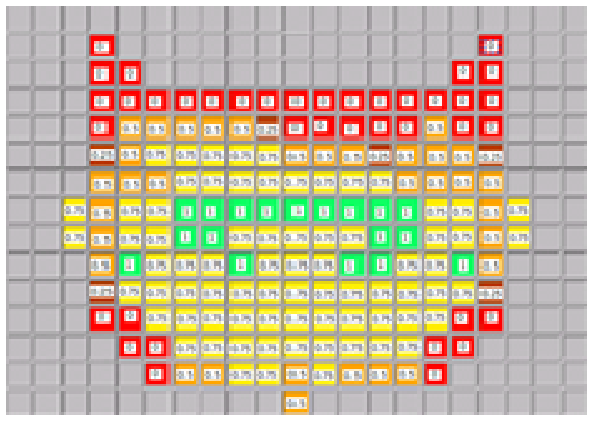

Fig.6 the score for the head before optimization
Table5 the meaning for each sign

\begin{tabular}{|c|c|c|}
\hline predicted results & sign & predicted score \\
\hline HIC $15<650$ & 1 & 1.000 \\
\hline $650 \leq \mathrm{HIC} 15<1000$ & 0.75 & 0.750 \\
\hline $1000 \leq \mathrm{HIC} 15<1350$ & 0.5 & 0.500 \\
\hline $\begin{array}{c}1350 \leq \mathrm{HIC} 15< \\
1700\end{array}$ & 0.25 & 0.250 \\
\hline $1700 \leq \mathrm{HIC} 15$ & 0 & 0.000 \\
\hline
\end{tabular}

The dotted line represents the WAD line, intersection line grid line for the impact test, total number of testing points is 170 .

According to the EuroNCAP pedestrian protection for head score calculated of 2013 version, each point scores are shown in figure 6 (Each sign represents the meaning shown in table 5).

According to the predicted score calculation of all the points, the total score of the original model is $91.5 \mathrm{pts}(1 * 18+0.75 * 74+0.5 * 33+0.25 * 6+0 * 33)$, and the score rate is $53.8 \%(91.5 / 170 * 100 \%)$. It ranks $12.9(24 * 0.538)$ out of 24 . Officially, to achieve EuroNCAP's 5 star level, the score of pedestrian protection must reach more than $60 \%$ of the total 36 pts, which is 21.6 pts. It is not very difficult to guarantee full 6 score of calf if you want to reach EuroNCAP's 5 standard which is about protection for pedestrian. The expected score of pedestrian head protection is 15.6pts(21.6-6), but there is still a wide gap between the predicted result for original model's head and the expected goal. We haven't took any consideration about such a high standard for pedestrian protection in the early development phase and the increasing requirement from the EurNCAP official, which are both main reasons for this phenomenon. So, to achieve EuroNCAP's 5 star requirement for pedestrian protection, we must improve the structure substantially. The main problems that remain in the original model list as follow: 
1) The hood inner panel stiffness is too high, it shows in the figure 7, the value of HIC15 in most hood regions is between 650 and 1000. According to the EuroNCAP2013, it needs further optimization to reduce the value of HIC15.

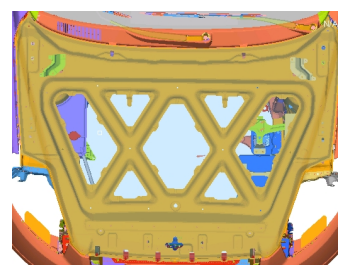

Fig.7 the hood inner panel

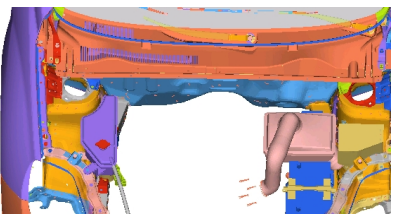

Fig.10 the ventilation panel

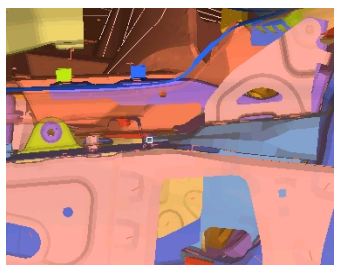

Fig.8 the hinge and support pole

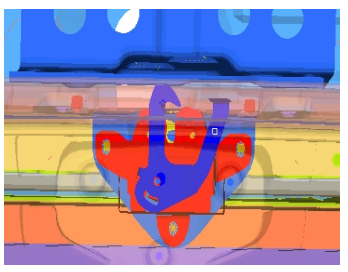

Fig.9 the hood lock

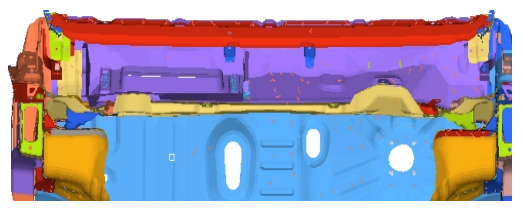

Fig.11 the glue install panels of windshield

2) The position of hood hinge is too high, the position of hood support pole is unreasonable, it shows in the figure 8 .

3) The position of hood lock is too high, it shows in the figure9.

4) Ventilation panel stiffness is too big, it shows in the figure 10.

5) The glue install panels of windshield and support stiffness is too big. It shows in the figure 11.

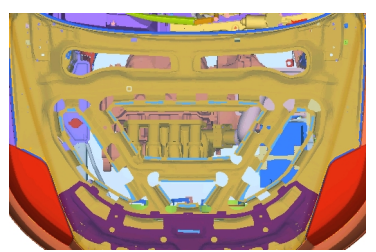

Fig.12 the new hood inner panel and strengthen panel

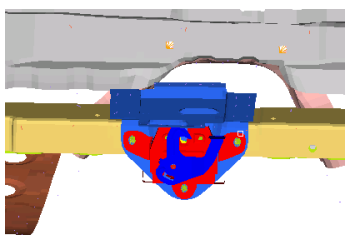

Fig.13 the new hood lock and strengthen panel

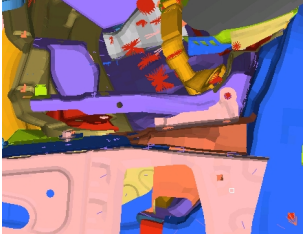

Fig.14the new hinge and strengthen panel

In this paper, we'll take an improved method as follows based on the result of optimization and experiment:

1) Redesign the hood inner panel, whose premise is to satisfy the hood performance of dent, stiffness, strength and shape, it's helpful to reduce its stiffness and strengthen the hood inner panel, it shows in the figure 12.

2) Readjust the position of the hood lock, redesign the lock strength panel, it shows in the figure 13 .

3) Redesign the position and shape of hood hinge, whose premise is to satisfy the performance and geometric kinematic relationships, it shows in the figure 14.

4) Redesign the wing panel support, it shows in the figure 15.

5) Redesign the shape and thickness of ventilation panel, it shows in the figure 16.

The result and score after the structure optimization shows in the figure 17(Meaning of each color shows on the chart 5). 


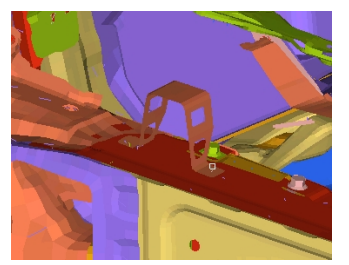

Fig.15 The new wing mounting bracket

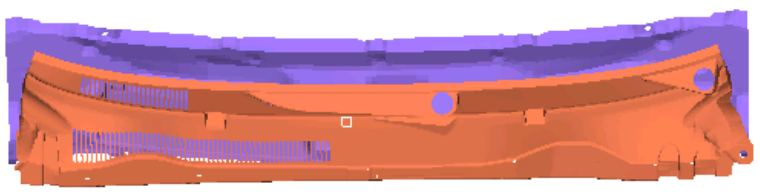

Fig.16 the new ventilation panel

The score rate after the optimization is 114.75 pts $(1 * 74+0.75 * 43+0.5 * 10+0.25 * 14+0 * 29)$, the rate is $67.4 \%$ $(114.5 / 170 * 100 \%)$, which is amounted to 24 value of 16.16 pts $(24 * 0.674)$, it meets the requirement in pedestrian protection for head performance, and there is a little security remainder.

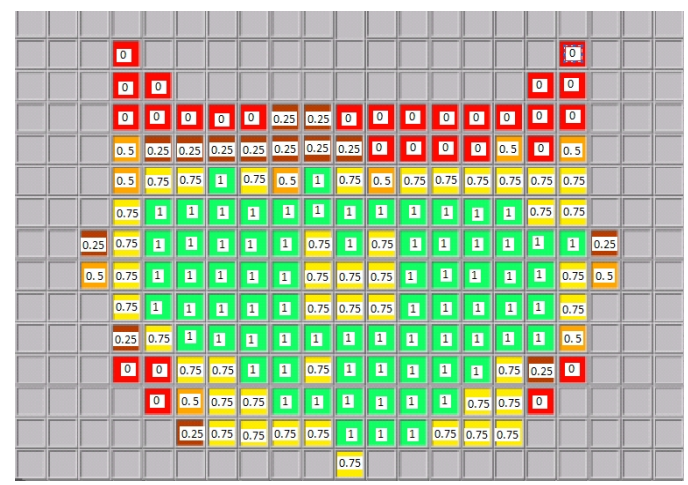

Fig.17 the score for the head after optimization

\section{Conclusion}

This paper is based on EuroNCAP2013's 5 star evaluation standard for pedestrian protection, head model impactor and structure model of the car front are used in the experiment. Combined with the results of the experiment, we use test analyze and simulation evaluation in pedestrian protection for head performance of SUV, then we take some improved design on the local body combined with the part structure feature of the collision points. The simulation result shows that the optimization project works more perfect in pedestrian protection for head performance.

\section{References}

[1]European New Car Assessment Program(EuroNCAP), Pedestrian Testing Protocol, Version $5.2,2010[\mathrm{~S}]$

[2]The national standard of the people's Republic of China, The Collision of Car to Pedestrian Protection, GB/T24550-2009[S]

[3] Zuofeng Pan, Yong Shan, Wei Ju. Study on Classification and Control Method for Pedestrians Head Impact Process

Support foundation project: Scientific research development foundation of Tianjin University of Technology and Education (KJ15-09) 